\title{
Soluble AxI Is a Novel Diagnostic Biomarker of Hepatocellular Carcinoma in Chinese Patients with Chronic Hepatitis B Virus Infection
}

\author{
Xiaoting Song, $M D^{1,2}$ \\ Ailu $\mathbf{W u}, \mathrm{MD}^{1,3}$ \\ Zhixiao Ding, MD2 \\ Shixiong Liang, $\mathrm{PhD}^{1}$ \\ Chunyan Zhang, $\mathrm{PhD}^{1}$
}

${ }^{1}$ Guangxi Medical University Cancer Hospital, Nanning, ${ }^{2}$ People's Hospital of Wudi County, Binzhou, ${ }^{3}$ The Affiliated Yantai Yuhuangding Hospital of Qingdao University Institution, Yantai, China

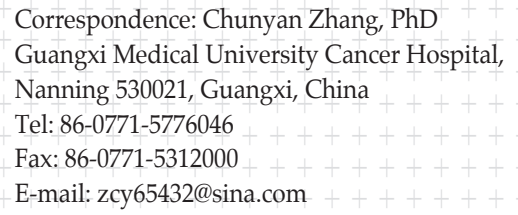

Co-correspondence: Shixiong Liang, $\mathrm{PhD}$ Guangxi Medical University Cancer Hospital, Nanning 530021, Guangxi, China

Tel: 86-0771-5335671

Fax: 86-0771-5312000

E-mail: shixliang@vip.sina.com

Received December 6, 2019

Accepted March 2, 2020

Published Online March 5, 2020

*Xiaoting Song, Ailu Wu, and Zhixiao Ding contributed equally to this work.

\begin{abstract}
Purpose
The purpose of this study was to evaluate the diagnostic value of soluble Axl (SAXl) in hepatocellular carcinoma (HCC) in comparison with serum $\alpha$-fetoprotein (AFP).
\end{abstract}

\section{Materials and Methods}

Eighty HCC patients, 80 liver cirrhosis patients (LC), 80 patients with hepatitis B virus (HBV) infection, and 80 healthy controls $(\mathrm{HC})$ were enrolled. sAxl levels were measured by an enzyme-linked immunosorbent assay, serum AFP levels were measured by an electrochemiluminescence immunoassay. Receiver operating characteristic (ROC) curves were used to evaluate diagnostic performances.

\section{Results}

The results show that levels of sAxl were high expression in patients with $\mathrm{HCC}(p<0.05)$, varied with disease state as follows: $\mathrm{HCC}>\mathrm{LC}>\mathrm{HC}>\mathrm{HBV}$. Logistic regression and ROC curve analysis identified the optimal cut-off for SAxl in differentiating all HCC and non-HCC patients was $1,202 \mathrm{pg} / \mathrm{mL}$ (area under the receiver operating characteristic [AUC], 0.888; $95 \%$ confidence interval [Cl], 0.852 to 0.924 ) with sensitivity $95.0 \%$, specificity $73.3 \%$. Furthermore, differential diagnosis of early HCC with non-HCC patients for sAxl showed the optimal cut-off was 1,202 pg/mL (AUC, 0.881; 95\% Cl, 0.831 to 0.931; sensitivity, 94.1\%; specificity, 73.3\%). Among AFP-negative HCC patients with non-HCC patients, the cut-off was $1,301 \mathrm{pg} / \mathrm{mL}$ (AUC, $0.898 ; 95 \% \mathrm{Cl}, 0.854$ to 0.942 ) with a sensitivity of $84.6 \%$, a specificity of $76.3 \%$. The optimal cut-off for SAxl in differentiating all $\mathrm{HCC}$ and chronic liver disease patients was $1,243 \mathrm{pg} / \mathrm{mL}$ (AUC, $0.840 ; 95 \% \mathrm{Cl}, 0.791$ to 0.888 ) with sensitivity $93.8 \%$, specificity $61.9 \%$. The combination of AFP and sAxl increased diagnostic value for HCC.

\section{Conclusion}

sAxl outperforms AFP in detecting HCC, especially in early HCC and in AFP-negative HCC. Combination sAxl with AFP improved the specificity for early HCC diagnosis. In summary, SAxl is a candidate serum marker for diagnosing HCC.

\section{Introduction}

Primary liver cancer (PLC) mainly includes hepatocellular carcinoma (HCC), intrahepatic cholangiocarcinoma (ICC) and the mixed type of HCC and ICC. HCC accounts for more than $90 \%$ of PLC. According to Cancer Statistics in China in 2015, the crude incidence rate of liver cancer was 26.92 per 100,000 population (males and females were 27.4 and 9.6 per
Key words

Hepatocellular carcinoma, Biomarker, Diagnosis, Soluble Axl 
leading to a median survival of $<1$ year [3]. Therefore, effective and accurate detection of HCC at an early stage is highly desired and may lead to more effective treatment and extent patient survival. The detection of serum biomarkers is the most promising approach and of tremendous importance as a non-invasive sampling.

Measurement of serum $\alpha$-fetoprotein (AFP) levels is the gold standard for high-risk patients to detect HCC at an early stage [4]. However, due to its low diagnostic accuracy with sensitivities ranging from $25 \%-65 \%$ and a specificity $74 \%$ $94 \%$, it is less than satisfactory as a widely used biomarker in the clinic $[5,6]$. Several new candidate biomarkers have been proposed to complement AFP and increased the accuracy of HCC detection, such as lectin-bound AFP (AFP-L3\%), des- $\gamma$-carboxyprothrombin, glypican3, Golgi protein-73, and Dickkopf-1. However, diagnostic values of all have controversial that limited clinical usefulness [6-9]. Thus, further research is necessary and urgent to identify additional novel candidates.

The receptor tyrosine kinase Axl belongs to the TAM family, which is comprised of Axl, tyro3, and Mer. Axl is activated by binding of ligand growtharrest specific protein six leading to dimerization and downstream signaling, which enhanced proliferation, survival, invasion, and metastasis $[10,11]$. Overexpression of Axl has been detected in many tumor types, in HCC as well [12-14]. Furthermore, Axl can be proteolytically processed, resulting in the release of an 80$\mathrm{kDa}$ soluble protein (sAxl) that can be observed in serum $[15,16]$. Recently, studies investigated that serum sAxl had better accuracy than AFP for diagnosis of early-stage HCC [17-19].

Guangxi province is a high incidence and mortality endemic area of HCC in China. Three thousand eight hundred members high-risk population cohort nested with case-control study method have been established since 2003. The major risk factor for HCC in the local area is still chronic hepatitis B virus (HBV) infection $[20,21]$. In this study, the primary aim was retrospectively to investigate the levels of peripheral serum sAxl and AFP with respecting to the sensitivity, specificity, area under the receiver operating characteristic (AUC) of each biomarker alone and a combination of two based on the serum samples of high-risk population cohort which comprised of HCC patients with HBV infection, patients with HBV-related liver cirrhosis (LC), patients with chronic HBV infection or healthy controls (HC). In addition, we evaluate the diagnostic accuracy of them for early-stage HCC.

\section{Materials and Methods}

\section{Patients and serum samples}

Serum samples from 80 patients with HBV infection HCC (HCC group), 80 patients with HBV-related liver cirrhosis (LC group), 80 patients with chronic hepatitis B virus infection (HBV group) or 80 healthy controls (HC group) were collected from Experimental Department of Guangxi Medical University Cancer Hospital, in China from October 2016 to July 2017. Serum samples were collected prior to therapeutic intervention. Taken venous blood samples in the early morning and allowed samples to clot for about 2 hours at room temperature before centrifuged for 15 minutes at $1,000 \times \mathrm{g}$. Remove serum and store samples at $-80^{\circ} \mathrm{C}$ until further assay. The diagnosis of primary HCC was based on guidelines of the American Association for the Study of Liver Diseases [22]. All patients were diagnosed either by histopathological results after surgical resection or by imaging findings (ultrasound, computed tomography or magnetic resonance) combined with AFP serum levels. HBV infection background was diagnosed on hepatitis B surface antigen. HCs were tested through routine physical examination. The exclusion criteria of HCs were based on alterations in serology, liver and kidney function and apparent chronic inflammatory diseases. Tumor stage was decided according to the Barcelona Clinic Liver Cancer (BCLC) [23]. In this study, early-stage HCC $(n=34)$ was defined as stage BCLC $0(n=1)$ and BCLC A $(n=33)$. Clinical information such as age, sex, tumor size, tumor number, vascular involvement, lymph node metastasis, HBV infection background, AFP level, and BCLC stage was obtained from a database of the Affiliated Tumor Hospital of Guangxi Medical University.

\section{Quantification of sAxl by enzyme-linked immunosor- bent assay and AFP by electrochemiluminescence immu- noassay}

Levels of human sAxl were measured by using a commercially available enzyme-linked immunosorbent assay (ELISA) kits (human sAxl ELISA kit, Cusabio, Wuhan, China) according to the manufacturer's instructions. Meanwhile, serum AFP was detected using electrochemiluminescence immunoassay (Elecsys and cobas e analyzers, Roche Diagnostics Mannheim, Germany) from the clinical laboratory of tumor hospital.

\section{Statistical analysis}

Statistical analysis was performed with IBM SPSS software ver. 22.0 (IBM Corp., Armonk, NY) and MedCalc software 
Table 1. Demographic and clinical characteristics of the study participants

\begin{tabular}{lcccc} 
Clinical characteristic & HCC & LC & HBV & HC \\
No. of patients & 80 & 80 & 80 & 50 \\
Sex (male/female) & $57 / 23$ & $62 / 18$ & $63 / 17$ & $51.6 \pm 9.7$ \\
Age, mean \pm SD $(\mathrm{yr})$ & $50.7 \pm 9.1$ & $49.0 \pm 11.9$ & $49.6 \pm 9.7$ & - \\
BCLC stage (0/A/B/C/D) & $1 / 33 / 14 / 28 / 4$ & - & $48 / 52$ & $80 / 0$ \\
AFP $(\leq 15 \mathrm{ng} / \mathrm{mL} />15 \mathrm{ng} / \mathrm{mL})$ & $26 / 54$ & $39 / 41$ & $80 / 0$ & $0 / 80$ \\
HBV status (positive/negative) & $80 / 0$ & $80 / 0$ & - \\
\hline
\end{tabular}

HCC, hepatocellular carcinoma; LC, liver cirrhosis; HBV, hepatitis B virus infection; HC, healthy controls; SD, standard deviation; BCLC, Barcelona Clinic Liver Cancer; AFP, $\alpha$-fetoprotein.

Table 2. sAxl and AFP levels of the study population

\begin{tabular}{lccc} 
Group & No. & sAxl $(\mathbf{p g} / \mathrm{mL})$ & AFP (ng/mL) \\
HCC & 80 & $2,020(1,546-2,526)$ & $162.3(6.4-3,514.5)$ \\
LC & 80 & $1,505(1,004-1,911)^{\mathrm{a})}$ & $16.7(3.5-79.7)^{\mathrm{a})}$ \\
HBV & 80 & $489(296-887)^{\mathrm{a}, \mathrm{b})}$ & $7.0(3.2-124.6)^{\mathrm{a}, \mathrm{b})}$ \\
HC & 80 & $678(469-893)^{\mathrm{a}, \mathrm{b}), \mathrm{c})}$ & $2.6(2.0-4.2)^{\mathrm{a}, \mathrm{b}) \mathrm{b}) \mathrm{d})}$ \\
AFP-positive HCC & 54 & $2,004(1,554-2,469)$ & $627.6(152.5-9,488.0)$ \\
AFP-negative HCC & 26 & $2,100(1,442-2,717)^{\mathrm{e})}$ & $3.9(3.0-6.4)^{\mathrm{f})}$ \\
BCLC (0+A) & 34 & $1,984(1,532-2,511)^{\mathrm{g})}$ & $91.1(4.0-613.2)^{\mathrm{g})}$ \\
BCLC (B) & 14 & $2,060(1,399-2,724)^{\mathrm{g})}$ & $682.4(68.0-8,446.3)^{\mathrm{g})}$ \\
BCLC (C) & 28 & $1,940(1,546-2,335)^{\mathrm{g})}$ & $229.0(6.0-9,620.0)^{\mathrm{g})}$ \\
\hline BCLC (D) & 4 & $2,524(1,784-3,817)^{\mathrm{g})}$ & $852.5(40.9-4,533.5)^{\mathrm{g})}$ \\
\hline
\end{tabular}

Values are presented as median (interquartile range). sAxl, soluble Axl; AFP, $\alpha$-fetoprotein; HCC, hepatocellular carcinoma; LC, liver cirrhosis; HBV, hepatitis B virus infection; HC, healthy controls; BCLC, Barcelona Clinic Liver Cancer. ${ }^{a)} \mathrm{p}<0.05$ (vs. HCC), b) $\mathrm{p}<0.05$ (vs. LC), ${ }^{c} \mathrm{p}>0.05$ (vs. HBV), ${ }^{\text {d) }} \mathrm{p}<0.05$ (vs. HBV), e) $\mathrm{p}>0.05$ (vs. AFP-positive HCC), ${ }^{\text {f) }} \mathrm{p}<0.05$ (vs. AFPpositive $\left.\mathrm{HCC}),{ }^{g}\right) \mathrm{p}>0.05$ (vs. BCLC(0+A)).

ver. 12.7.0 (Ostend, Belgium). Results were presented as medians (interquartile ranges). Nonparametric Kruskal-Wallis test was carried out for multiple comparisons and nonparametric two-sided Mann-Whitney test for comparisons between groups. Receiver operating characteristic (ROC) curves were utilized to evaluate diagnostic value. Binary logistic regression was generated to evaluate the performance of a combinatorial variable of two biomarkers. In this study, $\mathrm{p}<0.05$ or $\mathrm{p}<0.017$ (Bonferroni check) were considered statistically significant.

\section{Ethical statement}

This study was retrospectively conducted and approved by the Research Ethics Committee of the Guangxi Medical University Cancer Hospital. Informed consent was obtained from all inductees before the work began.

\section{Results}

\section{Patient characteristics}

A total of 320 participants were enrolled in our study. Demographic, clinical characteristics and classification of the study participants were shown in Table 1 . The most common cause of liver disease was HBV infection in group HCC, group LC, and group HBV. The HCC was developed from liver cirrhosis. There were no significant differences between each group in age and sex aspect.

\section{Levels of serum sAxl are significantly high in HCC pati- ents}

Serum of 320 participants was assessed for sAxl levels by ELISA. As shown in Table 2, the median levels of sAxl in 
A

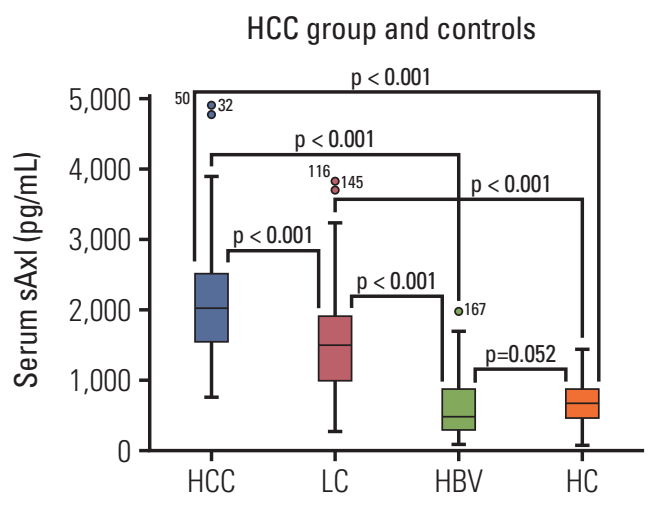

B

AFP-positive HCC group and AFP-negative HCC

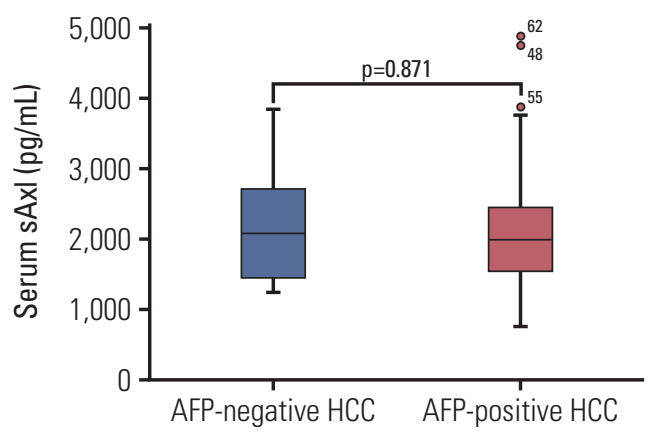

HCC defined in $B C L C$ stages with $0+A, B, C, D$

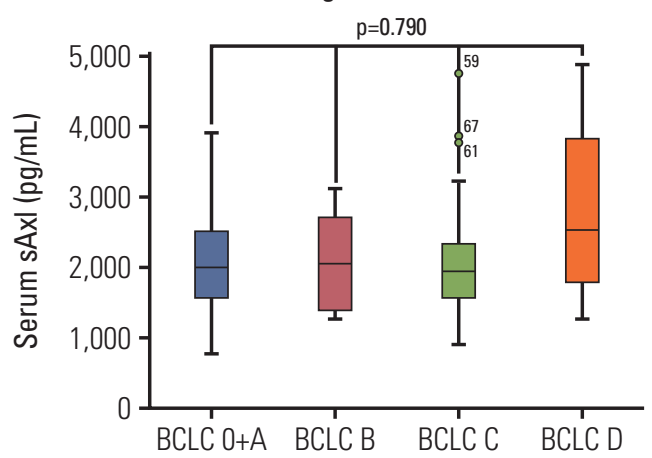

Fig. 1. Soluble Axl (sAxl) concentration in serum in hepatocellular carcinoma (HCC) patients. (A) Serum sAxl in HCC group and controls. (B) Serum sAxl in $\alpha$-fetoprotein (AFP)-positive HCC group and AFP-negative HCC. (C) Serum sAxl in HCC defined in Barcelona Clinic Liver Cancer stages with $0+\mathrm{A}, \mathrm{B}, \mathrm{C}, \mathrm{D}$. PLC, primary liver cancer; LC, liver cirrhosis; HBV, hepatitis B virus; HC, healthy control.

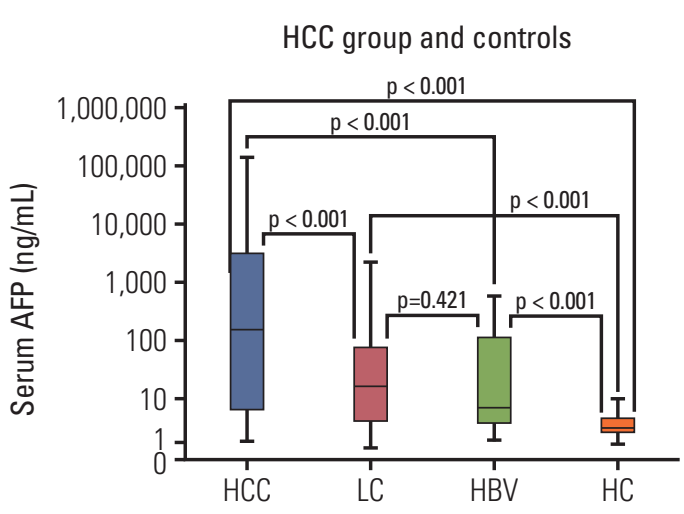

B

AFP-positive and AFP-negative HCC group

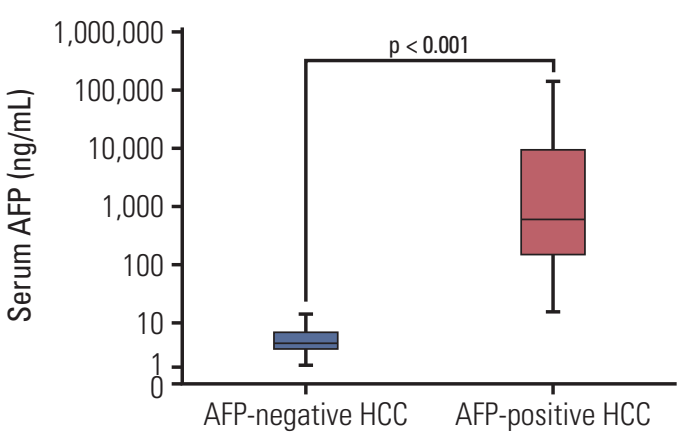

C

HCC defined in $B C L C$ stages with $0+A, B, C, D$

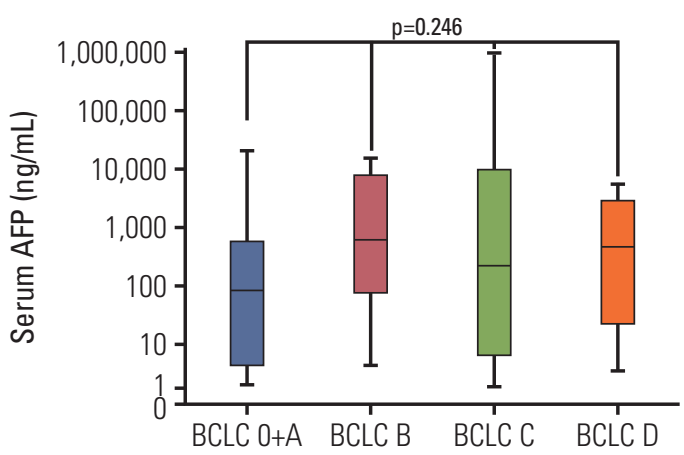

Fig. 2. $\alpha$-Fetoprotein (AFP) concentration in serum in hepatocellular carcinoma (HCC) patients. (A) Serum AFP in HCC group and controls. (B) Serum AFP in AFP-positive HCC group and AFP-negative HCC. (C) Serum AFP in HCC defined in Barcelona Clinic Liver Cancer stages with $0+A, B, C, D$. PLC, primary liver cancer; LC, liver cirrhosis; $\mathrm{HBV}$, hepatitis B virus; $\mathrm{HC}$, healthy control. 
Table 3. Diagnostic value of sAxl in detecting HCC

\begin{tabular}{|c|c|c|c|c|c|}
\hline Group & $\begin{array}{c}\text { AUC } \\
(95 \% \mathrm{CI})\end{array}$ & Sensiticity (\%) & Specificity (\%) & $\begin{array}{l}\text { Youden's } \\
\text { index }(\%)\end{array}$ & $\begin{array}{l}\text { Cut-off } \\
(\mathrm{pg} / \mathrm{mL})\end{array}$ \\
\hline \multicolumn{6}{|c|}{ HCC vs. non-HCC } \\
\hline sAxl & $0.888(0.852-0.924)$ & 95.0 & 73.3 & 68.3 & 1,202 \\
\hline AFP & $0.765(0.699-0.830)$ & 67.5 & 70.0 & 37.5 & 15,600 \\
\hline sAxl+AFP & $0.914(0.884-0.945)$ & 96.3 & 72.5 & 68.8 & - \\
\hline \multicolumn{6}{|l|}{ HCC vs. CLD } \\
\hline sAxl & 0.840 & 93.8 & 61.9 & 55.6 & 1,243 \\
\hline AFP & 0.703 & 66.3 & 61.9 & 28.1 & 21,480 \\
\hline sAxl+AFP & 0.875 & 83.8 & 73.8 & 57.5 & - \\
\hline \multicolumn{6}{|c|}{ Early HCC vs. non-HCC } \\
\hline sAxl & $0.881(0.831-0.931)$ & 94.1 & 74.2 & 67.5 & 1,202 \\
\hline AFP & $0.705(0.598-0.812)$ & 58.8 & 73.3 & 33.3 & 19,870 \\
\hline sAxl+AFP & $0.899(0.853-0.945)$ & 76.5 & 86.7 & 63.1 & - \\
\hline \multicolumn{6}{|c|}{ Early HCC vs. CLD } \\
\hline sAxl & $0.834(0.770-0.898)$ & 91.2 & 64.4 & 55.6 & 1,281 \\
\hline AFP & $0.636(0.518-0.755)$ & 58.8 & 61.3 & 20.1 & 19,870 \\
\hline sAxl+AFP & - & 100 & 82.5 & - & - \\
\hline \multicolumn{6}{|c|}{ AFP-negative HCC vs. non-HCC } \\
\hline sAxl & $0.898(0.854-0.942)$ & 84.6 & 76.3 & 60.9 & 1,301 \\
\hline \multicolumn{6}{|c|}{ AFP-negative HCC vs. CLD } \\
\hline sAxl & $0.849(0.785-0.912)$ & 73.1 & 74.4 & 47.5 & 1,555 \\
\hline
\end{tabular}

sAxl, soluble Axl; HCC, hepatocellular carcinoma; AUC, area under the receiver operating characteristic; CI, confidence interval; AFP, $\alpha$-fetoprotein; CLD, chronic liver disease, livercirrhosis+hepatitis B virus infection; non-HCC, livercirrhosis+ hepatitis B virus infection+healthy controls.

Table 4. Assessment of diagnostic value in HCC

\begin{tabular}{|c|c|c|c|}
\hline Group & p (AUC) & p (Se) & p (Sp) \\
\hline \multicolumn{4}{|l|}{ HCC vs. non-HCC } \\
\hline sAxl vs. AFP & 0.001 & $<0.001$ & $0.699^{a)}$ \\
\hline AFP vs. sAxl+AFP & $<0.001$ & $<0.001$ & - \\
\hline sAxl vs. sAxl+AFP & $<0.001$ & $>0.99$ & - \\
\hline \multicolumn{4}{|l|}{ HCC vs. CLD } \\
\hline sAxl vs. AFP & 0.003 & $<0.001$ & $>0.99$ \\
\hline AFP vs. sAxl+AFP & $<0.001$ & 0.011 & $0.023^{\mathrm{b})}$ \\
\hline sAxl vs. sAxl+AFP & 0.002 & 0.045 & $0.023^{b)}$ \\
\hline \multicolumn{4}{|c|}{ Early HCC vs. non-HCC } \\
\hline sAxl vs. AFP & 0.005 & 0.001 & $0.836^{\mathrm{b})}$ \\
\hline AFP vs. sAxl+AFP & $<0.001$ & 0.120 & 0.001 \\
\hline sAxl vs. sAxl+AFP & $0.059^{\mathrm{b})}$ & 0.040 & $<0.001$ \\
\hline \multicolumn{4}{|l|}{ Early HCC vs. CLD } \\
\hline sAxl vs. AFP & 0.006 & 0.002 & $0.563^{\mathrm{b})}$ \\
\hline AFP vs. sAxl+AFP & - & $<0.001$ & $<0.001$ \\
\hline sAxl vs. sAxl+AFP & - & $0.238^{\mathrm{b})}$ & $<0.001$ \\
\hline
\end{tabular}

HCC, hepatocellular carcinoma; AUC, area under the receiver operating characteristic; Se, sensitivity; Sp, specificity; nonHCC, livercirrhosis thepatitis B virus infection+healthy controls; sAxl, soluble Axl; AFP, $\alpha$-fetoprotein; CLD, chronic liver disease, livercirrhosis+hepatitis B virus infection. ${ }^{a)} \mathrm{p}>0.05,{ }^{\mathrm{b})} \mathrm{p}>0.017$. 
All HCC vs. LC, HBV, HC

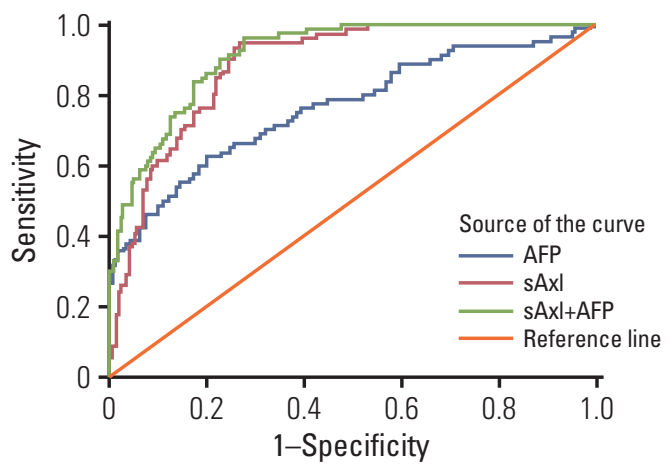

Early HCC vs. LC, HBV, HC

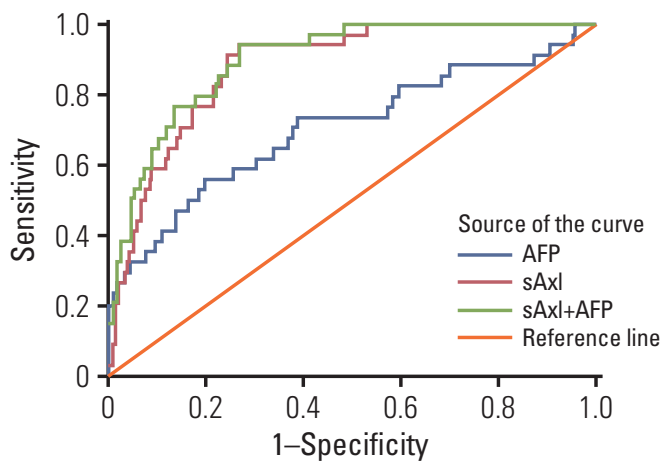

AFP-negative HCC vs. LC, HBV, HC

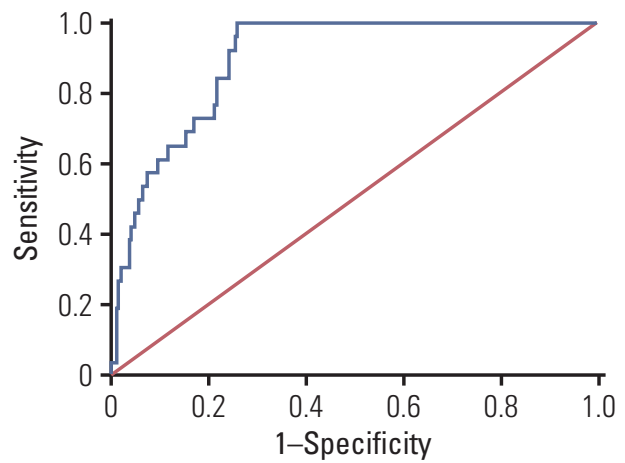

A

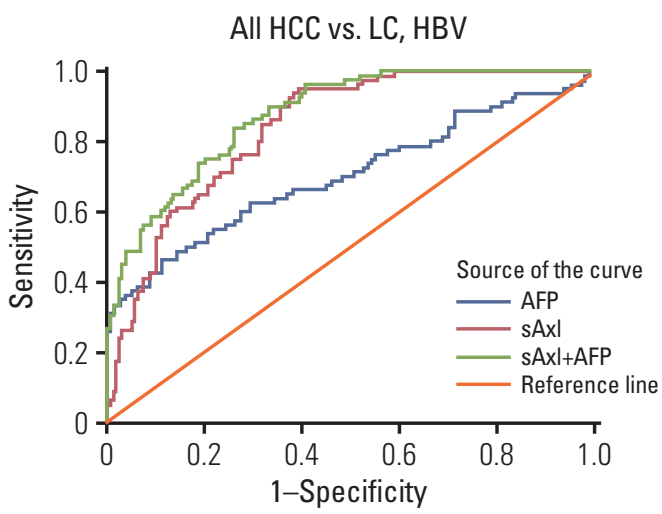

C

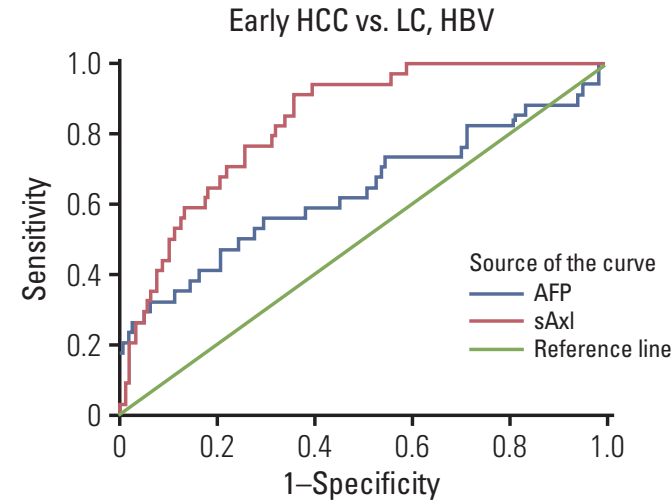

$\mathbf{F}$

$\mathbf{E}$

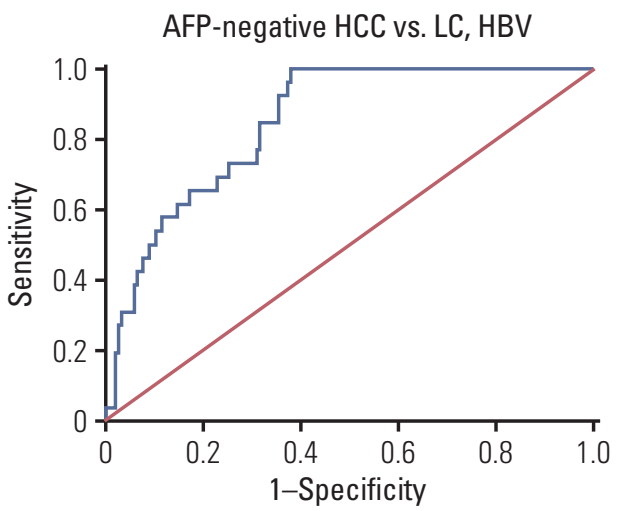

Fig. 3. Diagnostic performance of soluble Axl (sAxl) and $\alpha$-fetoprotein (AFP) in diagnosis of hepatocellular carcinoma (HCC) evaluated by receiver operating characteristic (ROC) curve. (A) ROC curve of sAxl in all HCC versus non-HCC. (B) ROC curve of sAxl in all HCC versus chronic liver disease (CLD). (C) ROC curve of sAxl in early HCC versus non-HCC. (D) ROC curve of sAxl in early HCC versus CLD. (E) ROC curve of sAxl in AFP-negative HCC versus non-HCC. (F) ROC curve of sAxl in AFP-negative HCC versus CLD. LC, liver cirrhosis; HBV, hepatitis B virus; HC, healthy control.

HCC patients was 2,020 pg/mL, which was significantly higher in comparison to LC patients $(1,505 \mathrm{pg} / \mathrm{mL}), \mathrm{HBV}$ patients $(489 \mathrm{pg} / \mathrm{mL})$, and HCs $(678 \mathrm{pg} / \mathrm{mL}, \mathrm{p}<0.05)$ (Fig. 1A). Although, the median concentration of healthy controls $(678 \mathrm{pg} / \mathrm{mL})$ was higher than that of HBV patients $(489 \mathrm{pg} / \mathrm{mL})$, there was no significant difference between them $(p>0.05)$. Similarly, significantly increased median concentration of AFP was found in HCC patients (162.3 
$\mathrm{ng} / \mathrm{mL})$, compared to LC patients $(16.7 \mathrm{ng} / \mathrm{mL})$, HBV patients $(7.0 \mathrm{ng} / \mathrm{mL})$, and healthy controls $(2.6 \mathrm{ng} / \mathrm{mL})($ all $\mathrm{p}<0.05$ ) (Fig. 2). HCCs were divided into AFP-positive HCC and AFP-negative HCC, according to $20.0 \mathrm{ng} / \mathrm{mL}$ of AFP levels. Significantly, the median concentration of sAxl was also increased in AFP-negative HCC and AFP-positive HCC (Fig. 1B). We also obtained a significant difference between AFP-negative HCC and non-HCC patients $(p<0.05)$. Also increased the median concentration of sAxl was observed in different BCLC stages. We did not detect any differences between each stage ( $p>0.05)$ (Fig. 1C).

\section{Diagnostic value of sAxl in detecting HCC}

To evaluate the diagnostic value of sAxl, an ROC curve was generated to identify the optimal cut-off, sensitivity, and specificity (Tables 3 and 4). sAxl exhibited an AUC of 0.888 with a sensitivity of $95 \%$ and a specificity of $73.3 \%$ at a cutoff of 1,202 pg/mL for all HCC vs. non-HCC (Fig. 3A). When the analysis was limited to all HCC vs. chronic liver disease (CLD), the resulting AUC was 0.840 with $93.8 \%$ sensitivity and $61.9 \%$ specificity at a cut-off of $1,243 \mathrm{pg} / \mathrm{mL}$ (Fig. 3B). Interestingly, sAxl outperformed AFP in diagnostic performance of AUC and sensitivity in both HCC vs. non-HCC or CLD (all $\mathrm{p}<0.017$ ). Next, we also assessed the diagnostic performance of a combination of two markers. Results showed that combined analysis improved AUC than sAxl alone (all $\mathrm{p}<0.017)$, but significance was not found in sensitivity and specificity (all $\mathrm{p}>0.017$ ) (Fig. 3A and B).

\section{Diagnostic value of sAxl in early HCC and AFP-negative HCC}

We next investigated diagnostic performance of sAxl in early HCC (BCLC $0+A, n=34)$ and AFP-negative HCC (AFP $\leq 20 \mathrm{ng} / \mathrm{mL}$ ). In differential diagnosis of early HCC versus non-HCC, sAxl also displayed higher accuracy (AUC, 0.881) and sensitivity (94.1\%) as compared to AFP (AUC, 0.705; sensitivity, 58.8\%) (Tables 3 and 4, Fig. 3C). Combined detection of two markers improved specificity than $\mathrm{sAxl}$ alone $(\mathrm{p}<$ 0.017). Binary logistic regression was further performed to create new variable predicted the probability for early HCC versus CLD, variables of AFP was not in the equation as $p>$ 0.05 . Results were similarly found in this part that sAxl had a better AUC and sensitivity than AFP ( $p<0.017)$ (Fig. 3D). Parallel combined detection indicated a higher specificity than markers alone $(\mathrm{p}<0.017)$. Furthermore, sAxl was able to distinguish AFP-negative HCC from non-HCC with an AUC of $0.898,84.6 \%$ sensitivity, and $76.3 \%$ specificity (Fig. 3E). Similarly, when AFP-negative HCC were compared with CLD, sAxl exhibited an AUC of 0.849 with a sensitivity of $73.1 \%$ and a specificity of $74.4 \%$ (Fig. 3F).

\section{Discussion}

HCC remains a kind of lethal malignant tumor worldwide and is still difficult to diagnostic in the early stage. One-third of HCC patients are AFP-negative [24,25]. It is greatly needed to identify a novel serum marker for HCC. sAxl levels have been used as a potential marker of HCC in current reports [17-19]. Therefore, we examined levels of sAxl to test and verify the diagnosis power in HCC. The results demonstrated that sAxl yielded an AUC of 0.888 with $95.0 \%$ sensitivity and $73.3 \%$ specificity in differentiating HCC from non-HCC that AUC of 0.840 with $93.8 \%$ sensitivity and $61.9 \%$ specificity in differentiating HCC from CLD. Furthermore, sAxl gave a similarly differentiating power in the diagnosis of early HCC and both AFP-negative HCC. Moreover, the combined measurement of sAxl and AFP can further improved diagnostic value for the detection of HCC. Our results suggest that sAxl is a potentially useful marker for HCC.

Our study showed that levels of serum sAxl of HCC were significantly higher compared to LC, HBV, and HC. It may reflect that $\mathrm{SAxl}$ can be used to monitor disease progression in patients with HCC and be distinguished HCC from LC, HBV, or HC. Dengler et al. [18] suggested that sAxl was predominantly released by myofibroblasts during fibrosis progression supported by the fact that sAxl was strongly released in six out of seven liver myofibroblasts cell lines under investigation and increased sAxl levels in advanced fibrosis and cirrhosis was observed significantly when compared to healthy controls. Staufer et al. [26] showed that sAxl was an accurate biomarker for advanced fibrosis and LC in comparison to established non-invasive fibrosis markers such as enhanced liver fibrosis test and transient elastography (Fibroscan). Further study showed that sAxl played an important role in the progression of liver fibrosis $[27,28]$. High levels of sAxl was also detected in LC group in our study which may reflect that sAxl was interrelated with fibrosis or LC. A great deal of studies indicated that Axl is relevant with tumorigenesis such as proliferation, survival, invasion, and metastasis $[10-12,29,30]$. Therefore, the molecular mechanism needs to be further explored.

Although Reichl et al. [17] had shown that the expression of sAxl in HCC was higher than in breast, ovarian and colorectal cancer, nevertheless sAxl as a biomarker has low specificity in the diagnosis of HCC in our study. It is necessary to combined sAxl with specific biomarkers such as AFP and other tumor markers to overcome the limitation. On the other hand, we have not found that sAxl have connection with BCLC stage. We prospect that a tumor marker could meet the following criteria such as specific overexpression in tumor but not in normal cells, rare expression in human normal tissues except in embryonic tissue and can be easily 
detected. Further exploration is needed for our study.

HBV infection is the main reason for HCC in China [21]. But patters were different from other areas, such as the United States, Europe, and Japan. Results were inspired of sAxl in diagnosis HCC with HBV in our study. It is significant to explore diagnostic power in non-HBV-related HCC.

Furthermore, 34 early-stage HCC $(42.5 \%)$ which were defined into BCLC $0+\mathrm{A}$ and 26 AFP-negative HCC (32.5\%) were recruited in the study. The sample size and proportion were small. Therefore, the diagnostic power of sAxl in earlystage HCC or AFP-negative HCC was indeed.

Our study belongs to cross-sectional and retrospective in nature, but it lacks randomization and short-term follow-up.
We intend to do a prospective study with local characteristics in Guangxi province to validate diagnosis value of sAxl and further explore the relationship of sAxl with clinical characters.

In conclusion, our study indicated that sAxl has differentiating power to diagnosis HCC, especially for early-stage HCC or AFP-negative HCC. Therefore, sAxl is a promising biochemical marker of HCC.

\section{Conflicts of Interest}

Conflict of interest relevant to this article was not reported.

\section{References}

1. Zheng RS, Sun KX, Zhang SW, Zeng HM, Zou XN, Chen R, et al. Report of cancer epidemiology in China, 2015. Zhonghua Zhong Liu Za Zhi. 2019;41:19-28.

2. Bray F, Ferlay J, Soerjomataram I, Siegel RL, Torre LA, Jemal A. Global cancer statistics 2018: GLOBOCAN estimates of incidence and mortality worldwide for 36 cancers in 185 countries. CA Cancer J Clin. 2018;68:394-424.

3. Abraham JA, Golubnitschaja O. Time for paradigm change in management of hepatocellular carcinoma: is a personalized approach on the horizon? Per Med. 2016;13:455-67.

4. Tsuchiya N, Sawada Y, Endo I, Saito K, Uemura Y, Nakatsura T. Biomarkers for the early diagnosis of hepatocellular carcinoma. World J Gastroenterol. 2015;21:10573-83.

5. Daniele B, Bencivenga A, Megna AS, Tinessa V. Alpha-fetoprotein and ultrasonography screening for hepatocellular carcinoma. Gastroenterology. 2004;127(5 Suppl 1):S108-12.

6. Chen H, Chen S, Li S, Chen Z, Zhu X, Dai M, et al. Combining des-gamma-carboxyprothrombin and alpha-fetoprotein for hepatocellular carcinoma diagnosing: an update meta-analysis and validation study. Oncotarget. 2017;8:90390-401.

7. Zhao Y, Gao Q, Pei L, Wang C, Jin L, Liao F. Current status and future prospects of biomarkers in the diagnosis of hepatocellular carcinoma. Int J Biol Markers. 2017;32:e361-9.

8. Kim SU, Park JH, Kim HS, Lee JM, Lee HG, Kim H, et al. Serum Dickkopf-1 as a biomarker for the diagnosis of hepatocellular carcinoma. Yonsei Med J. 2015;56:1296-306.

9. Lim TS, Kim DY, Han KH, Kim HS, Shin SH, Jung KS, et al. Combined use of AFP, PIVKA-II, and AFP-L3 as tumor markers enhances diagnostic accuracy for hepatocellular carcinoma in cirrhotic patients. Scand J Gastroenterol. 2016;51:344-53.

10. Han J, Tian R, Yong B, Luo C, Tan P, Shen J, et al. Gas6/Axl mediates tumor cell apoptosis, migration and invasion and predicts the clinical outcome of osteosarcoma patients. Biochem Biophys Res Commun. 2013;435:493-500.

11. Wu X, Ma W, Zhou Q, Yan H, Lim ZF, Huang M, et al. AXLGAS6 expression can predict for adverse prognosis in non- small cell lung cancer with brain metastases. J Cancer Res Clin Oncol. 2017;143:1947-57.

12. Reichl P, Dengler M, van Zijl F, Huber H, Fuhrlinger G, Reichel C, et al. Axl activates autocrine transforming growth factor-beta signaling in hepatocellular carcinoma. Hepatology. 2015;61:930-41.

13. Xu J, Jia L, Ma H, Li Y, Ma Z, Zhao Y. Axl gene knockdown inhibits the metastasis properties of hepatocellular carcinoma via PI3K/ Akt-PAK1 signal pathway. Tumour Biol. 2014;35: 3809-17.

14. Liu J, Wang K, Yan Z, Xia Y, Li J, Shi L, et al. Axl expression stratifies patients with poor prognosis after hepatectomy for hepatocellular carcinoma. PLoS One. 2016;11:e0154767.

15. Ekman C, Stenhoff J, Dahlback B. Gas6 is complexed to the soluble tyrosine kinase receptor Axl in human blood. J Thromb Haemost. 2010;8:838-44.

16. Dengler M, Huber H, Muller CJ, Zellmer A, Rauch P, Mikulits W. Accurate determination of soluble Axl by enzyme-linked immunosorbent assay. Assay Drug Dev Technol. 2016;14:54350.

17. Reichl P, Fang M, Starlinger P, Staufer K, Nenutil R, Muller P, et al. Multicenter analysis of soluble Axl reveals diagnostic value for very early stage hepatocellular carcinoma. Int J Cancer. 2015; 137:385-94.

18. Dengler M, Staufer K, Huber H, Stauber R, Bantel H, Weiss $\mathrm{KH}$, et al. Soluble Axl is an accurate biomarker of cirrhosis and hepatocellular carcinoma development: results from a large scale multicenter analysis. Oncotarget. 2017;8:46234-48.

19. Reichl P, Mikulits W. Accuracy of novel diagnostic biomarkers for hepatocellular carcinoma: an update for clinicians (review). Oncol Rep. 2016;36:613-25.

20. Guo J, Pan LH, Li YX, Yang XD, Li LQ, Zhang CY, et al. Efficacy of triclosan-coated sutures for reducing risk of surgical site infection in adults: a meta-analysis of randomized clinical trials. J Surg Res. 2016;201:105-17.

21. Wang YT, Chen TY, Zhu J, Jiao YC, Qu CF. Primary preven- 
tion by hepatitis B vaccine on liver cancer in high incidence area of China. Zhonghua Yu Fang Yi Xue Za Zhi. 2018;52: 402-8.

22. Kudo M. Evidence and consensus on the management of hepatocellular carcinoma: update 2015. Oncology. 2015;89 Suppl 2:1-3.

23. Weinmann A, Koch S, Sprinzl M, Kloeckner R, SchulzeBergkamen H, Duber C, et al. Survival analysis of proposed BCLC-B subgroups in hepatocellular carcinoma patients. Liver Int. 2015;35:591-600.

24. Li J, Cheng ZJ, Liu Y, Yan ZL, Wang K, Wu D, et al. Serum thioredoxin is a diagnostic marker for hepatocellular carcinoma. Oncotarget. 2015;6:9551-63.

25. Shen Q, Fan J, Yang XR, Tan Y, Zhao W, Xu Y, et al. Serum DKK1 as a protein biomarker for the diagnosis of hepatocellular carcinoma: a large-scale, multicentre study. Lancet Oncol. 2012;13:817-26.

26. Staufer K, Dengler M, Huber H, Marculescu R, Stauber R,
Lackner $C$, et al. The non-invasive serum biomarker soluble Axl accurately detects advanced liver fibrosis and cirrhosis. Cell Death Dis. 2017;8:e3135.

27. Smirne C, Rigamonti C, De Benedittis C, Sainaghi PP, Bellan $\mathrm{M}$, Burlone ME, et al. Gas6/TAM signaling components as novel biomarkers of liver fibrosis. Dis Markers. 2019;2019: 2304931.

28. Tutusaus A, de Gregorio E, Cucarull B, Cristobal H, Areste C, Graupera I, et al. A functional role of GAS6/ TAM in nonalcoholic steatohepatitis progression implicates AXL as therapeutic target. Cell Mol Gastroenterol Hepatol. 2019;9:349-68.

29. Xu MZ, Chan SW, Liu AM, Wong KF, Fan ST, Chen J, et al. AXL receptor kinase is a mediator of YAP-dependent oncogenic functions in hepatocellular carcinoma. Oncogene. 2011; 30:1229-40.

30. Paccez JD, Vogelsang M, Parker MI, Zerbini LF. The receptor tyrosine kinase Axl in cancer: biological functions and therapeutic implications. Int J Cancer. 2014;134:1024-33. 